\title{
Digital Radiography/Computed Tomography of Medical Devices
}

\section{James Troedel ${ }^{1}$}

${ }^{1 .}$ Baxter International, Round Lake, IL USA.

As technology advances, medical device architecture and manufacturing processes increase in complexity and so too do the requirements regarding quality control, validation and generally demonstrating understanding of the device or process. Many tools typically used for their analysis, such as disassembly, cross-sectioning, or laser or optical scanning, have limitations due to accuracy, complexity, time or cost issues. For example, the intricate internal geometries of a small device could be altered or destroyed during cross-sectioning or disassembly. And while laser or optical scanning will allow for accurate measurements, fixturing can be a challenge amongst a wide material range and internal structure imaging is not possible. Digital radiography (DR) paired with computed tomography (CT) represents all of these tools without their limitations in one package thereby providing a higher level of understanding of the part or process in a fraction of the time. It is a powerful non-destructive imaging technique that allows for dynamic, magnified x-ray viewing of internal components as well as extensive volumetric post processing analyses of the reconstructed $3 \mathrm{D}$ volume.

Commonly referred to as micro-CT in reference to the ability to achieve single micrometer resolution and also known as industrial CT, it works by emitting x-rays through a sample as it rotates 360 degrees while capturing hundreds to thousands of digital radiographs, or x-rays. The radiographs are then reconstructed into a 3D image through which it can be sectioned or sliced to reveal the internal layout. The resulting data can then be used to identify defects, view surface interfaces and part fitment, obtain dimensional measurements and image internal functions. Post processing of the data using volumetric software for metrology, CAD comparison or reverse engineering activities can also be applied.

The technique was pioneered in the early 1970's and since has been used extensively by a variety of industries such as aerospace and automotive. Those industries were early adopters of the technology due to the ability to inspect critical safety-related components. By comparison, the pharmaceutical and medical device industries arrived on scene a bit later but were quick to realize the value of the technique as the complexity of their products grew as did the quality and regulatory requirements. The principles of micro-CT are similar to medical CT with a few differences being an approximately $100 \mathrm{X}$ increase in resolution between medical $\mathrm{CT}$ and micro-CT and fewer concerns regarding the $\mathrm{x}$-ray intensity and exposure times when using micro-CT. By far the biggest downside to industrial CT is the cost which increases proportional to the largest sample size that can be scanned. Small benchtop units start around $\$ 300 \mathrm{~K}$ and some investment in human capital must be made as there is a sharp learning curve with regards to scanning technique and development of post processing tools.

Digital radiography by itself is a useful tool that can provide real-time video and images without the time associated with a typical CT scan and reconstruction which is about an hour in most cases. A device can be viewed, with geometric magnification of 1-40X depending on sample size, immediately without the requirement for cross-sectioning or other sample preparation while it moves to provide perspective in the 2D environment. Functioning internal components can also be viewed if the device can be set in motion or activated by remote control. Determinations regarding function and fit can then be made simultaneously. Examples of real time digital radiography use include visualizations of circuit 
traces, fluid delivery pump mechanisms and activation of reconstitution devices. The ability to scan a device or part while it functions in conjunction with multiple CT scans can also yield 4D video, as shown in Figure 1.

In most cases, CT is also used with DR to provide better accuracy and resolution. The 3D perspective and ability to scroll through slices in the model improves detectability such as the voids observed within the part shown in Figure 2. The part was scanned for dimensional comparison to 2D drawings when voids were discovered during routine reconstruction. Also, CT processing times can be lowered but still reveal valuable data. Figure 3 shows the results of 15 minute processing time (scan and reconstruction) that shows the desired dimensional information relative to specifications and the morphology of the welds.

In addition to being a unique imaging tool, $\mathrm{DR} / \mathrm{CT}$ also has numerous engineering applications since it can provide volumetric data to assist with activities such as mold qualification and first article inspection or produce a polygon file for production a 3D printed model of the device. Considering the broad range of uses and the level of understanding it provides, this technology can be a crucial tool for many investigations.
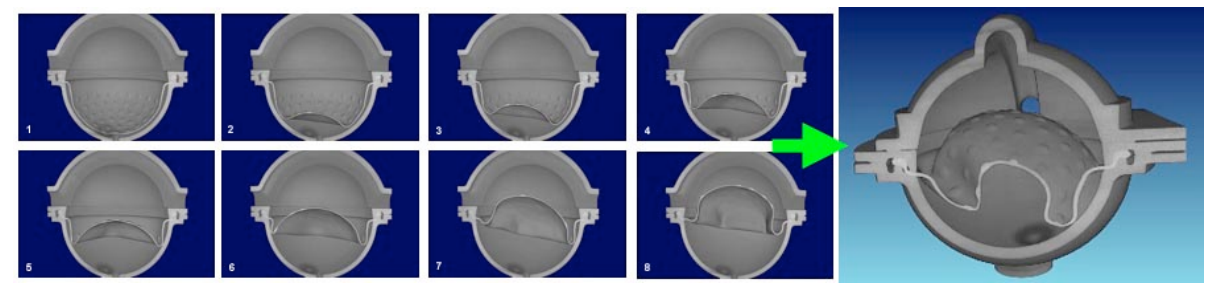

Figure 1. Screenshots of eight CT scans captured during different stages of diaphragm movement within a fluid pump that were then assembled into a 4D video.



Figure 2. For these bottle adaptor images, the left image shows the surfaced part for creation of a stereolithograph (.stl) file. During reconstruction of the $3 \mathrm{D}$ volume voids were discovered within the material as shown in the image of the adaptor on the right which is sliced in two planes.

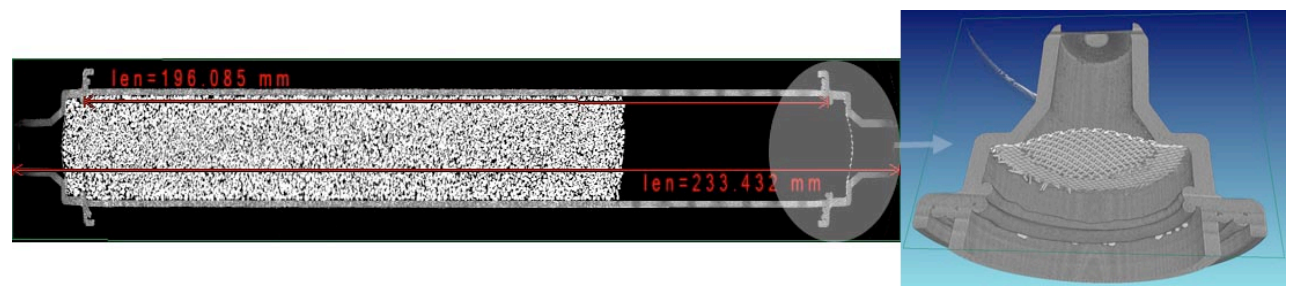

Figure 3. Results of a 15 minute CT scan of cartridge device where both dimensional information relative to specifications from the $2 \mathrm{D}$ radiographs and weld morphology from the $3 \mathrm{D}$ reconstruction were acquired. 